\title{
Recovery from Copperhead Snake Envenomation: Role of Age, Sex, Bite Location, Severity, and Treatment
}

\author{
Eric J. Lavonas ${ }^{1,2,3}$ D - Randy I. Burnham ${ }^{1}$ • John Schwarz ${ }^{1}$. Eugenia Quackenbush ${ }^{4}$ - Brandon Lewis ${ }^{5}$. \\ S. Rutherfoord Rose ${ }^{6}$. Spencer Greene ${ }^{7}$. Eric A. Toschlog ${ }^{8} \cdot$ Nathan P. Charlton $^{9} \cdot$ Michael E. Mullins ${ }^{10}$. \\ Richard Schwartz ${ }^{11}$. David Denning ${ }^{12} \cdot$ Kapil Sharma $^{13} \cdot$ Kurt Kleinschmidt $^{13} \cdot$ Sean P. Bush $^{14} \cdot$ Victoria E. Anderson $^{1}$. \\ Adit A. Ginde ${ }^{3}$. Charles J. Gerardo ${ }^{15}$
}

Received: 12 April 2019 / Revised: 23 July 2019 / Accepted: 19 August 2019 /Published online: 3 September 2019

(C) American College of Medical Toxicology 2019

\begin{abstract}
Introduction Few data exist to understand the recovery phase of pit viper envenomation. A recently published placebo-controlled clinical trial affords this opportunity. The purpose of this study is to examine the time course of recovery from copperhead snake (Agkistrodon contortrix) envenomation patients managed with and without the use of antivenom, stratified by age, sex, anatomic site of envenomation, initial severity of envenomation, and geographic region.

Methods This is a post-hoc subgroup analysis of data from a multi-center double-blinded clinical trial of Fab antivenom (FabAV) vs. placebo. Outcomes were the Patient-Specific Functional Scale (PSFS) score at 3, 7, 10, and 14 days after envenomation. Least-squares mean PSFS score curves were calculated for each subgroup, and repeated measures ANOVA was used to estimate between-group comparisons.

Results Seventy-two subjects were included, of whom 44 received FabAV. Males demonstrated better overall recovery than females (model predicted PSFS score 6.18 vs 4.99 ; difference 1.19 ; $95 \%$ CI 0.12 to $2.25 ; p=0.029$ ). No sex difference was found in response to FabAV. Overall recovery and effect of FabAV were similar in adult vs adolescent patients, patients with
\end{abstract}

This study has not been presented previously.

Supervising Editor: Mark B. Mycyk, MD

Electronic supplementary material The online version of this article (https://doi.org/10.1007/s13181-019-00733-y) contains supplementary material, which is available to authorized users.

Eric J. Lavonas eric.lavonas@dhha.org

1 Rocky Mountain Poison and Drug Center, Denver Health and Hospital Authority, Denver, CO, USA

2 Department of Emergency Medicine, Denver Health and Hospital Authority, Denver, CO, USA

3 Department of Emergency Medicine, University of Colorado School of Medicine, Aurora, CO, USA

4 Department of Emergency Medicine, University of North Carolina School of Medicine, Chapel Hill, NC, USA

5 Texas A\&M Health Science Center, College Station, TX, USA

6 Department of Emergency Medicine, Virginia Commonwealth University, Richmond, VA, USA

7 Henry J. N. Taub Department of Emergency Medicine, Baylor College of Medicine, Houston, TX, USA

8 Department of Surgery, Brody School of Medicine at East Carolina University, Greenville, NC, USA

9 Division of Medical Toxicology, University of Virginia, Charlottesville, VA, USA

10 Division of Emergency Medicine, Washington University School of Medicine, St. Louis, MO, USA

11 Department of Emergency Medicine and Hospital Services, Medical College of Georgia, Augusta, GA, USA

12 Department of Surgery, Marshall Health, Huntington, WV, USA

13 Department of Emergency Medicine, University of Texas Southwestern Medical Center, Dallas, TX, USA

14 Department of Emergency Medicine, Brody School of Medicine at East Carolina University, Greenville, NC, USA

15 Division of Emergency Medicine, Department of Surgery, Duke University School of Medicine, Durham, NC, USA 
upper vs lower extremity envenomation, and patients with initially mild vs moderate envenomation signs. Analysis by geographic location was not successful due to ANOVA mode instability.

Conclusions Male victims of copperhead snake envenomation demonstrate slightly better recovery than females, but response to Fab antivenom overall is similar across all subgroups studied.

Keywords Envenomation $\cdot$ Snake $\cdot$ Agkistrodon $\cdot$ Antivenins $\cdot$ Snake $\cdot$ Fab $\cdot$ Copperhead

\section{Introduction}

Approximately 5000-9000 people seek treatment in US emergency departments each year due to snake envenomation, nearly half of whom are envenomated by a single species, the copperhead snake (Agkistrodon contortrix) [1, 2]. Because the clinical manifestations of copperhead envenomation tend to be less severe than rattlesnake envenomation, copperhead victims were excluded from the preclinical studies of the ovine Fab snake antivenom (Crotalidate Polyvalent Immune Fab (ovine), BTG International Inc., West Conshohocken PA; hereafter, FabAV), but copperhead envenomation has been an approved indication for FabAV administration since the drug's initial approval in October, 2000 [3-5]. Subsequent studies showed lasting limb morbidity following copperhead envenomation $[6,7]$.

Data from the US National Poison Data System suggest that FabAV use is less common in copperhead envenomation victims (36\% of whom received FabAV in 2007) than rattlesnake victims (55\%), although these proportions are increasing [6]. This is likely because copperhead envenomation patients rarely develop life-threatening systemic effects, such as hypotension, neurotoxicity, severe defibrinogenation, or severe thrombocytopenia [8-11]. Although the previous, equine-derived antivenom had serious problems with acute hypersensitivity reactions and serum sickness that restricted its use to more serious envenomation cases, FabAV is generally safe and well-tolerated [12-15]. However, antivenoms are expensive. The author of a 2004 editorial in Annals of Emergency Medicine opined, "the routine use of [FabAV] in the patient with mild to moderate local swelling alone should be based on evidence of improved outcome... In addition, it is important that any potential benefit be determined in a prospective controlled manner to obtain the best evidence for cost-effective treatment of these patients." [16]. A randomized, double-blinded clinical trial demonstrated that patients receiving FabAV recover more quickly than placebo recipients, although spontaneous recovery in the placebo-treated subjects closes the gap by 28 days after treatment [17]. Subsequent analyses have shown that early treatment with FabAV produces better improvement than delayed treatment with FabAV and that the duration of opioid analgesic use is shorter in patients who receive FabAV $[18,19]$.

Neither the original studies of short-term response to FabAV nor the more recent trial of the effect of FabAV on limb recovery has examined whether the natural history of snake envenomation or response to therapy differs between different groups of patients. This study seeks to determine whether important differences exist in overall recovery and/ or the response to FabAV between men and women, between patients bitten on the upper extremity vs. the lower extremity, between patients with initially mild or moderate local tissue venom injury, and between patients envenomated in different geographic locations within the range of the copperhead snake.

\section{Methods}

This is a post-hoc analysis of subgroup data collected in the course of a randomized clinical trial (NCT\# 01864200), the methods and main results of which have been published [17]. In this study, 74 patients who had been envenomated by copperhead snakes were randomized to receive FabAV or visually identical placebo. Details about study sites, patient selection, inclusion and exclusion criteria, FabAV dosing, study assessments, and the complete trial protocol have been previously published [17]. For this study, we used the modified intent-totreat population, all subjects who received study medication and had at least one post-treatment visit. One of the original study subjects received study drug and then withdrew from the study to attend to a family emergency without completing any post-treatment assessments. One patient was determined to have falsified his history of snake envenomation. Missing data were imputed using the last observation carried forward method (LOCF).

As in the original, this study uses results of the PatientSpecific Functional Scale (PSFS), a patient-oriented outcome measure that has been widely validated in other musculoskeletal disorders and has been specifically validated in copperhead snake envenomation [20-26]. The Minimal Clinically Important Difference (MCID) of the PSFS in copperhead snake envenomation is 1.0 points [27]. Ratings for the PSFS were collected by in-person assessment performed 3, 7, and 14 days after envenomation, and telephonically on day 10 . Telephonic administration of the PSFS has recently been validated [28]. Missing data were imputed using the last observation carried forward method. All analyses were conducted using SAS® (v. 9.4, Cary, NC). 
Raw mean PSFS scores with standard errors were calculated for each subgroup for each time point and presented graphically.

In order to use all available data to preserve statistical power, for tests of significance, a repeated measured ANOVA model of the mean PSFS was implemented, controlling for treatment effects over time. Sex, age stratification (adolescent/adult), anatomic bite location (lower/upper extremity), severity of envenomation at the time of enrollment (mild/moderate), study site, and treatment group (FabAV/placebo) were treated as categorical variables, and the PSFS was treated as a continuous variable. Follow-up times of 3, 7, 10, and 14 days were treated as equidistant within the model. The assumption of independent errors for ANOVA is violated when repeated measurements are serially correlated; therefore, an autoregressive error structure of order one was used to account for stronger correlations of measurements closer in time and weaker correlations of measurements further apart in time.

We used backwards stepwise regression to identify any factors that were independently associated with either overall recovery or response to treatment. In the base model, all subgroup variables were added into the model, along with their interaction terms by treatment group. The subgroup variable with the largest $p$ value greater than 0.1 was used to remove the subgroup fixed effect along with any interaction terms involving the subgroup at each iteration. This iterative process continued until a final model with all remaining subgroups

Table 1 Demographics and envenomation characteristics

\begin{tabular}{|c|c|c|c|}
\hline Case characteristics & $\begin{array}{l}\text { FabAV-treated } \\
\text { patients }(N=44)\end{array}$ & $\begin{array}{l}\text { Placebo-treated } \\
\text { patients }(N=28)\end{array}$ & $\begin{array}{l}\text { Total-treated } \\
\text { patients }(N=72)\end{array}$ \\
\hline \multicolumn{4}{|l|}{ Age (year) } \\
\hline Mean (SD) & $44.1(18.08)$ & $42.0(17.42)$ & $43.3(17.73)$ \\
\hline Range & $(12.0,86.0)$ & $(13.0,69.0)$ & $(12.0,86.0)$ \\
\hline \multicolumn{4}{|l|}{ Age strata, No. (\%) } \\
\hline Adolescent & $3(6.8 \%)$ & $4(14.3 \%)$ & $7(9.7 \%)$ \\
\hline Adult & $41(93.2 \%)$ & $24(85.7 \%)$ & $65(90.3 \%)$ \\
\hline \multicolumn{4}{|l|}{ Sex, No. $(\%)$} \\
\hline Male & $22(50.0 \%)$ & $15(53.6 \%)$ & $37(51.4 \%)$ \\
\hline \multicolumn{4}{|l|}{ Race, No. (\%) } \\
\hline White & $39(88.6 \%)$ & $24(85.7 \%)$ & $63(87.5 \%)$ \\
\hline Black & $2(4.5 \%)$ & $2(7.1 \%)$ & $4(5.6 \%)$ \\
\hline Asian & $1(2.3 \%)$ & $0(0.0 \%)$ & $1(1.4 \%)$ \\
\hline Other & $2(4.5 \%)$ & $2(7.1 \%)$ & $4(5.6 \%)$ \\
\hline \multicolumn{4}{|l|}{ Ethnicity, No. (\%) } \\
\hline Hispanic or Latino & $3(6.8 \%)$ & $4(14.3 \%)$ & $7(9.7 \%)$ \\
\hline Not Hispanic or Latino & $41(93.2 \%)$ & $24(85.7 \%)$ & $65(90.3 \%)$ \\
\hline \multicolumn{4}{|l|}{ Anatomic location, No. (\%) } \\
\hline Lower Extremity & $29(65.9 \%)$ & $16(57.1 \%)$ & $45(62.5 \%)$ \\
\hline Upper Extremity & $15(34.1 \%)$ & $12(42.9 \%)$ & $27(37.5 \%)$ \\
\hline \multicolumn{4}{|l|}{ Severity at enrollment, No. (\%) } \\
\hline Mild & $39(88.6 \%)$ & $25(89.3 \%)$ & $64(88.9 \%)$ \\
\hline Moderate & $5(11.4 \%)$ & $3(10.7 \%)$ & $8(11.1 \%)$ \\
\hline \multicolumn{4}{|l|}{ Study site, No. (\%) } \\
\hline Duke University, Durham, NC & $15(34.1 \%)$ & $11(39.3 \%)$ & $26(36.1 \%)$ \\
\hline University of North Carolina, Chapel Hill, NC & $12(27.3 \%)$ & $4(14.3 \%)$ & $16(22.2 \%)$ \\
\hline St. Joseph Regional Health Center, Bryan, TX & $8(18.2 \%)$ & $4(14.3 \%)$ & $12(16.7 \%)$ \\
\hline Virginia Commonwealth University Medical Center, Richmond, VA & $2(4.5 \%)$ & $2(7.1 \%)$ & $4(5.6 \%)$ \\
\hline East Carolina University Brody School of Medicine, Greenville, NC & $2(4.5 \%)$ & $1(3.6 \%)$ & $3(4.2 \%)$ \\
\hline Barnes-Jewish Hospital, St Louis, MO & $1(2.3 \%)$ & $1(3.6 \%)$ & $2(2.8 \%)$ \\
\hline Other sites & $4(9.1 \%)$ & $5(17.9 \%)$ & $9(12.5 \%)$ \\
\hline
\end{tabular}

Other sites enrolling subjects were Ben Taub General Hospital and Texas Children's Hospital, Houston, TX; Marshall Health Medical Center, Huntington, WV; University of Virginia Medical Center, Charlottesville, CA; and Augusta University Medical Center, Augusta, GA

FabAV Crotalidae Polyvalent Immune Fab (ovine) 
was found such that all subgroup $p$ values were less than 0.1 . Visual residual diagnostics were performed on the final model to review model assumptions and assess model fit. The least square means for these subgroups of significance were summarized, and the difference was calculated with a corresponding $95 \%$ confidence interval and $p$ value.

A sensitivity analysis was also completed on the final model, reinserting the subject who falsified his snake envenomation. The sensitivity analysis was performed to see if the significance of least square means or model fit drastically changed from the addition of one outlier subject.

\section{Results}

Demographic and envenomation characteristics of the 72 included subjects are presented in Table 1. Missing data were imputed using LOCF for 5/288 (1.7\%) of observations.

Subgroup results for sex, age, anatomic bite location, and initial severity are presented in Figs. 1, 2, 3, and 4. The same information is presented with a direct comparison by treatment group, in On-Line Resource 1. As expected in an

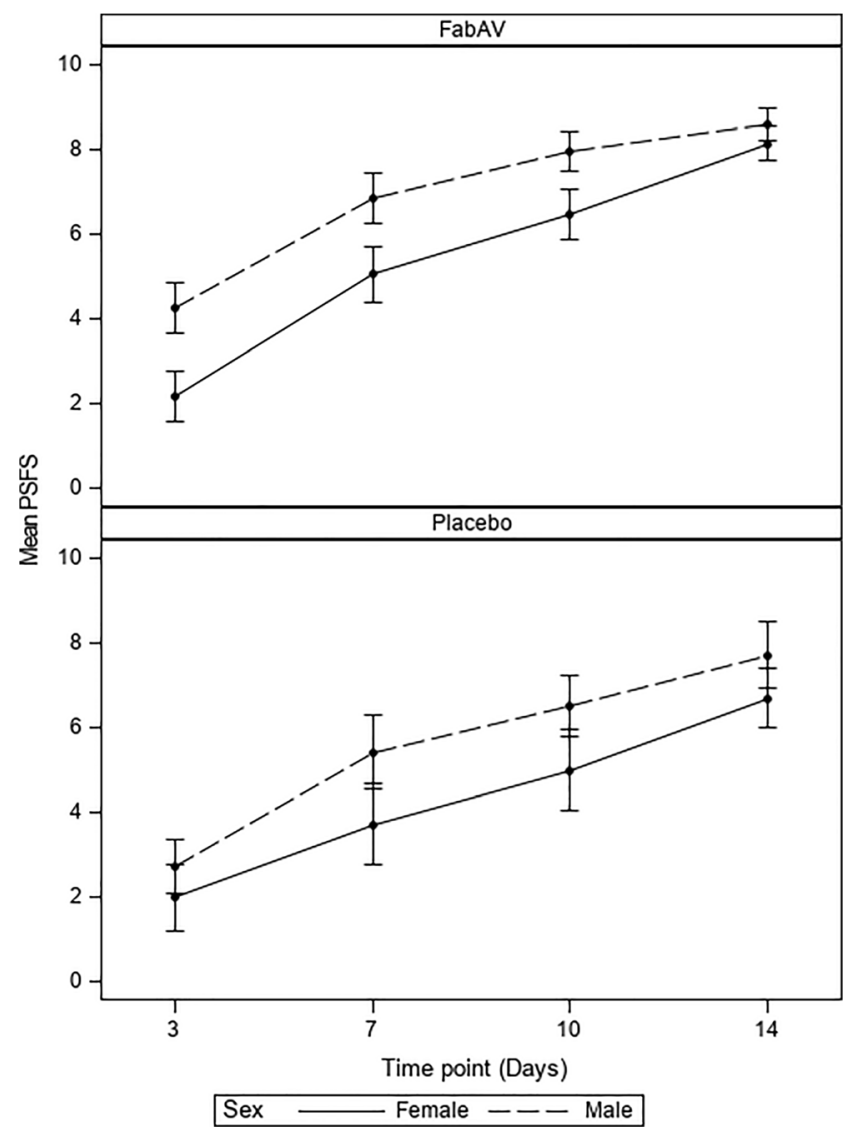

Fig. 1 Mean scores on the Patient-Specific Functional Scale during recovery from copperhead snake envenomation, by sex and treatment. Raw mean Patient-Specific Functional Scale (PSFS) scores and standard error for each time point (days after treatment) and treatment group. FabAV Fab antivenom analysis of small subgroups, the estimates of functional recovery (raw mean PSFS scores) were not significantly different for any given subgroup and time point, though with a single exception (adolescents at day 7), the results tended towards better recovery in FabAV-treated subjects.

ANOVA model convergence could not be obtained when including a random effect for treatment study site, including logical geographic combinations of sites, so site was removed from the model. Raw data for the highest-enrolling study sites are presented in On-Line Resource 2.

In the tests for significance, age, initial severity of envenomation, and anatomic bite location were all found not to be associated with either overall recovery or response to FabAV therapy and were sequentially removed from the model. Sex, treatment group, the interaction of treatment group, and visit number remained significantly related to PSFS score in the final model, as shown on Table 2. Although non-significant, the interaction of treatment group and sex was retained in the final model to control for differences within treatment for each sex in predicting the least-squared means in the final model.

The final model showed that men had a higher predicted PSFS (6.18 vs 4.99; difference 1.19; 95\% CI for difference

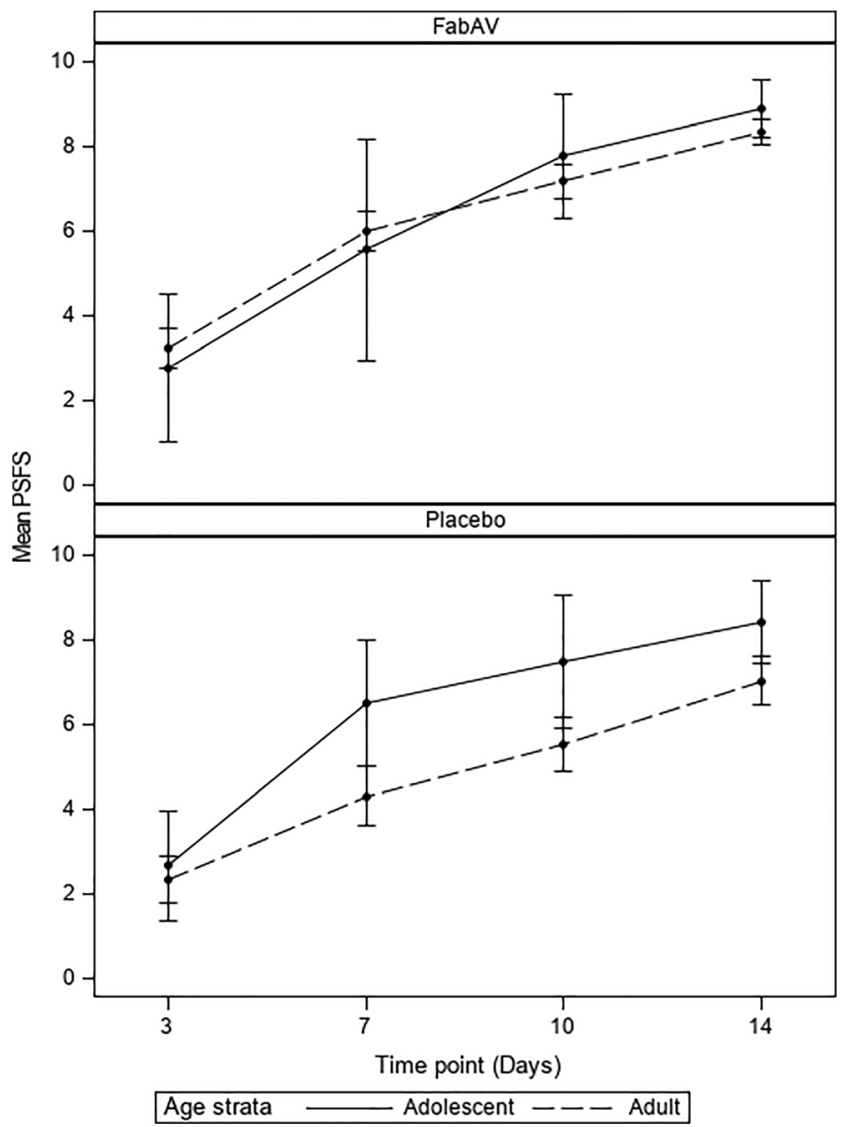

Fig. 2 Mean scores on the Patient-Specific Functional Scale during recovery from copperhead snake envenomation, by age group and treatment. Raw mean Patient-Specific Functional Scale (PSFS) scores and standard error for each time point (days after treatment) and treatment group. FabAV Fab antivenom 


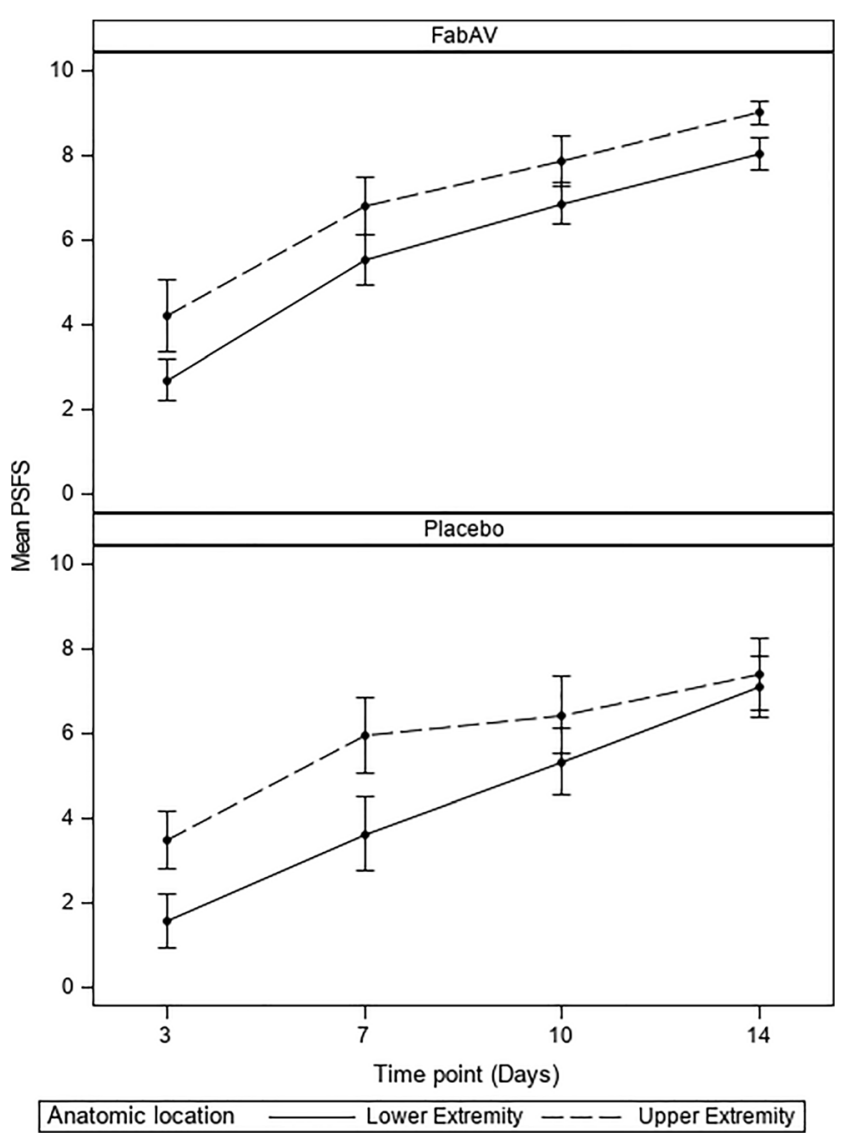

Fig. 3 Mean scores on the Patient-Specific Functional Scale during recovery from copperhead snake envenomation, by envenomated extremity and treatment. Raw mean Patient-Specific Functional Scale (PSFS) scores and standard error for each time point (days after treatment) and treatment group. FabAV Fab antivenom

0.12 to $2.25 ; p=0.029)$, after controlling for treatment effects and time. There was no significant difference in the response to FabAV treatment between men and women.

In the sensitivity analysis, re-insertion of the factitious envenomation patient resulted in no significant change to the main model results.

\section{Discussion}

In patients with mild to moderate severity copperhead snake envenomation, treatment with FabAV leads to more rapid recovery of limb function than placebo therapy [15]. In this study, it appears that short-term recovery (3-14 days after treatment) was similar for adolescents, patients bitten on the upper extremity, and patients with mild initial swelling, compared with adults and those with lower extremity bites or moderate initial swelling. Men had better functional status than women overall. No differences in the response to FabAV therapy were found based on age, sex, bitten extremity, and initial severity.

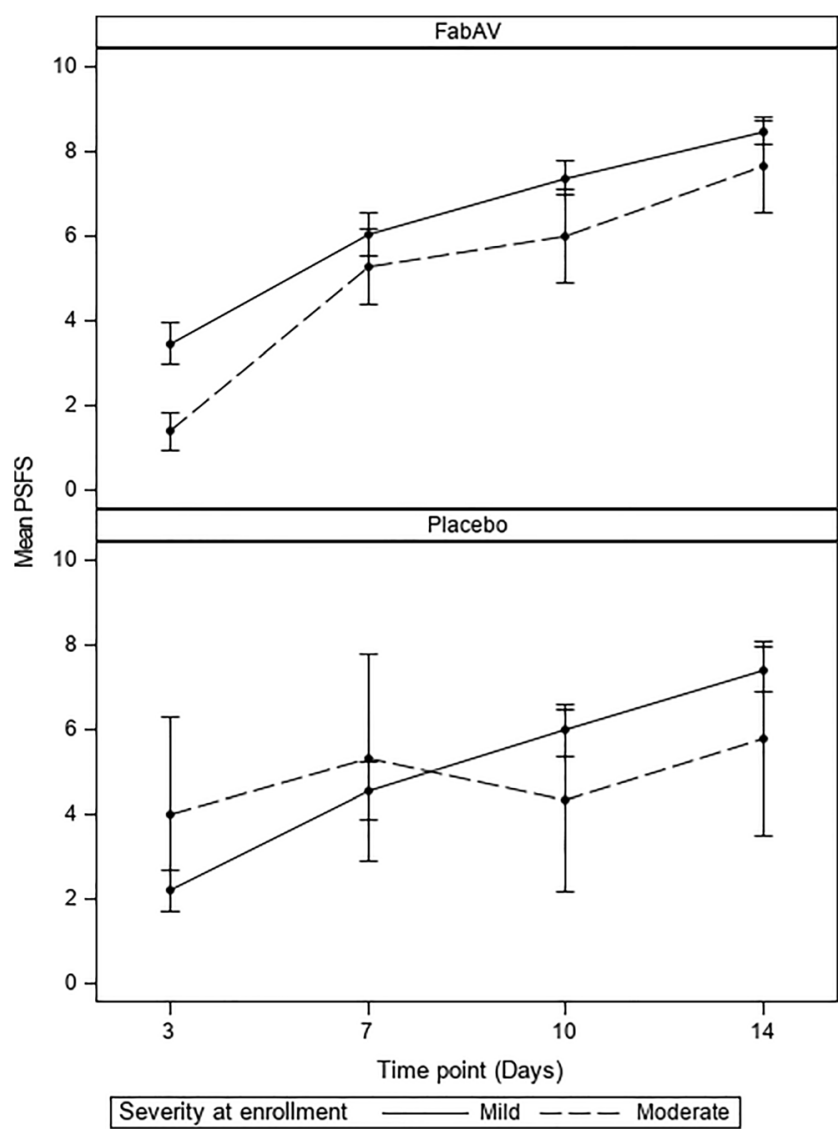

Fig. 4 Mean scores on the Patient-Specific Functional Scale during recovery from copperhead snake envenomation, by initial severity of envenomation and treatment. Raw mean Patient-Specific Functional Scale (PSFS) scores and standard error for each time point (days after treatment) and treatment group. FabAV Fab antivenom

The small number of subjects in several subgroups makes it possible that true differences were not apparent. This is particularly true for adolescents and patients with moderate initial envenomation severity, where subgroups were very small. Random-cause variation affecting small subgroup data is also apparent in our results.

In our multivariate model, we were unable to test for differences based on the geographic location where the envenomation occurred. The interaction between treatment group and time was significant, indicating that patients who received FabAV improved more rapidly than placebo-treated patients.

Table 2 Tests of fixed effects from the backward model selection

\begin{tabular}{lc}
\hline Model effect & $P$ value $(F$-test $)$ \\
\hline Treatment & 0.0292 \\
Treatment $\times$ visit number & $<.0001$ \\
Sex & 0.0290 \\
Treatment $\times$ sex & 0.7665 \\
\hline
\end{tabular}


To our knowledge, no previous manuscript has examined variations in crotaline envenomation presentation or response to therapy by sex, bitten extremity, or severity on initial presentation. Although many stand-alone pediatric manuscripts exist, we are not aware of publications comparing outcomes in adolescent to adult populations.

These data are useful to the front-line clinician in two ways. First, this study did not identify a group that was likely to respond differently to FabAV administration than another; thus, patient-centered shared decision making incorporating discussions of benefit, risk, and cost can proceed in a similar fashion for all patients. Second, this study did not identify a group with a differentially better or worse prognosis for recovery; all patients with mild to moderate copperhead envenomation can be told that return to normal, on average, takes $2-$ 4 weeks, with faster recovery in men and in patients treated with FabAV [16].

It is particularly surprising, and counterintuitive, that no significant difference in limb recovery was found in patients with mild vs. moderate severity envenomation signs prior to treatment. This is likely due to lack of statistical power given the small number of patients (8) with moderate initial envenomation. Though defined by objective criteria, the definition of "mild" envenomation used in this study encompassed some patients who would be considered to have "moderate" local tissue swelling in some other systems.

It is interesting to note that in one Texas center, patients receiving placebo appeared to have better outcomes than FabAV-treated subjects (Fig. A1). Given the small number of subjects involved, the lack of biologic plausibility for harm to recovery of limb function from antivenom (as opposed to no effect), and the large number of comparison performed, we suspect that this was a random result (i.e., epiphenomenon). However, regional differences in envenomation outcomes and response to FabAV are a worthwhile topic for future research.

An alternative explanation for the observed differences between men and women is that sex and/or gender affect responses to the PSFS instrument. Although we can find on indication that such differences exist, it does not appear that the question has been formally studied.

\section{Limitations}

This is a secondary analysis of prospectively collected clinical trial data. The overall dataset is relatively small, and subgroup analyses are underpowered and highly susceptible to beta error. Put another way, it is possible that important differences in either overall outcomes or the benefit of antivenom exist based on patient age, bite severity, envenomated limb, or across the geographic range of the copperhead snake that were not detected in this analysis. In order to fit a linear model, this study did not measure the phase of recovery from day 14 , when the difference between FabAV and placebo-treated subjects was greatest, and day 28 , when average recovery between FabAV-treated and placebo patients converges. The overall magnitude of benefit from FabAV, a 1.2 point difference on the PSFS metric on day 14, is close to the MCID. Imprecision in the estimate of the treatment effect is much greater in subgroup analyses than in the larger study, particularly when a subgroup is small, and in virtually all of these subgroup analyses, it is possible that treatment benefit is too small to be clinically meaningful to the patient. Although the repeated measures ANOVA modeling methods we used takes the number of comparisons into account when calculating statistical significance, the model cannot compensate for our multiple approaches to attempt to evaluate study site as a variable.

\section{Conclusion}

Recovery during the first 14 days after copperhead snake envenomation appears to be greater among men than women, but response to Fab antivenom is similar. No differences in recovery were seen between adolescents and adults, between victims bitten on the upper vs. lower extremity, and between those with initially mild or moderate venom effects.

Sources of Funding This work was supported by a grant from BTG International, Inc. to the Rocky Mountain Poison and Drug Center, and by NIH/NCATS Colorado CTSI Grant Number UL1 TR002535. Contents are the authors' sole responsibility and do not necessarily represent official NIH views, nor those of BTG International.

\section{Compliance with Ethical Standards}

Conflict of Interest This clinical trial and the subsequent subgroup analysis were funded by BTG International, Inc. In addition, the Denver Health and Hospital Authority (EJL, RJB, JS, and VJA) has research, consulting, and call center agreements with BTG International, Inc., and has completed research arrangements with the manufacturer of a competing antivenom. Duke University (CJG) has received research funding from BTG for projects beyond the current trial. Dr. Green has received income from BTG for professional consulting. No other author has received personal funds or has other conflicts to disclose.

\section{References}

1. O'Neil ME, Mack KA, Gilchrist J. Epidemiology of non-canine bite and sting injuries treated in U.S. emergency departments, 20012004. Pub Health Rep. 2007;122:764-75.

2. Gummin DD, Mowry JB, Spyker DA, Brooks DE, Fraser MO, Banner W. 2016 annual report of the American Association of Poison Control Centers' National Poison Data System (NPDS): 34th annual report. Clin Toxicol. 2017;55:1072-252. 
3. Dart RC, Seifert SA, Carroll L, Clark RF, Hall E, Boyer-Hassen LV, et al. Affinity-purified, mixed monospecific crotalid ovine fab for the treatment of crotalid venom poisoning. Ann Emerg Med. 1997;30:33-9.

4. Dart RC, Seifert SA, Boyer LV, Clark RF, Hall E, McKinney P, et al. A randomized multicenter trial of Crotalinae polyvalent immune fab (ovine) antivenom for the treatment for crotaline snakebite in the United States. Arch Intern Med. 2001;161:2030-6.

5. Epstein JS. Approval Letter - CroFab. US Food and Drug Administration, October 2, 2000. Available at: https://www.fda. gov:80/FDAgov/BiologicsBloodVaccines/BloodBloodProducts/ A p provedProducts/LicensedProductsB LAs / FractionatedPlasmaProducts/ucm 117586 .htm. Accessed 16 Nov 2018.

6. Seifert SA, Boyer LV, Benson BE, Rogers JJ. AAPCC database characterization of native US venomous snake exposures, 2001 2005. Clin Toxicol (Phila). 2009;47(4):327-335.

7. Lavonas EJ, Kerns WP, Gerardo CJ, Richardson W, Whitlow KS, Berkoff DJ. Long-term limb function outcomes after copperhead snakebite. Ann Emerg Med. 2008;52:S141-2.

8. Lavonas EJ, Gerardo CJ, O'Malley GO, Arnold TC, Bush SP, Banner $\mathrm{W}$, et al. Initial experience with crotaline fab antivenom in the treatment of copperhead (Agkistrodon contortrix) snakebite. Ann Emerg Med. 2004;43:200-6.

9. Lavonas EJ, Kokko J, Schaeffer TH, Mlynarchek SL, Bogdan GM, Dart RC. Short-term outcomes following fab antivenom therapy for severe crotaline snakebite. Ann Emerg Med. 2011;57:128-37.

10. Yin S, Kokko J, Lavonas E, Mlynarchek S, Bogdan G, Schaeffer T. Factors associated with difficulty achieving initial control with Crotalidae polyvalent immune fab antivenom in snakebite patients. Acad Emerg Med. 2011;18:46-52.

11. Gerardo CJ, Vissoci JR, Brown MW, Bush SP. Coagulation parameters in copperhead compared to other Crotalinae envenomation: secondary analysis of the $\mathrm{F}\left(\mathrm{ab}^{\prime}\right) 2$ versus fab antivenom trial. Clin Toxicol. 2017;55:109-14.

12. Schaeffer TH, Khatri V, Zolot L, Lavonas EJ. Incidence of immediate hypersensitivity reaction and serum sickness following administration of Crotalidae polyvalent immune fab antivenom - a metaanalysis. Acad Emerg Med. 2012;19:121-31.

13. Kleinschmidt KC, Ruha A-M, Campleman S, Brent J, Wax P, on behalf of the ToxIC North American Snakebite Registry Group. Acute adverse events associated with the administration of Crotalidae polyvalent immune Fab antivenom within the North American Snakebite Registry. Clin Toxicol. 2018;13:309-20.

14. Khobrani M, Huckleberry Y, Boesen KJ, Aljabri A, Alharthi M, Patanwala AE. Incidence of allergic reactions to Crotalidae polyvalent immune fab. Clin Toxicol. 2018;57:164-7. https://doi.org/10. 1080/15563650.2018.1504956.

15. Mullins ME, Gerardo CJ, Bush SP, et al. Adverse Events in the Efficacy of Crotalidae Polyvalent Immune Fab Antivenom vs Placebo in Recovery from Copperhead Snakebite Trial. South Med J. 2018. https://doi.org/10.14423/SMJ.0000000000000902.

16. Caravati EM. Copperhead bites and Crotalidae polyvalent immune fab (ovine): routine use requires evidence of improved outcomes. Ann Emerg Med. 2004;43:207-8.
17. Gerardo CJ, Quackenbush E, Lewis B, Rose SR, Greene S, Toschlog EA, et al. The efficacy of fab antivenom versus placebo plus optional rescue therapy on recovery from copperhead snake envenomation: a randomized, double-blind, placebo-controlled clinical trial. Ann Emerg Med. 2017;70:233-44.

18. Anderson VE, Gerardo CJ, Rapp-Olsson M, Bush SP, Mullins ME, Greene S, Toschlog EA, Quackenbush E, Rose SR, Schwartz RB, Charlton NP, Lewis B, Kleinschmidt KC, Sharma K, Lavonas EJ. Early administration of Fab antivenom resulted in faster limb recovery in copperhead snake envenomation patients. Clin Toxicol (Phila). 2019; 57(1):25-30.

19. Freiermuth CE, Lavonas EJ, Anderson VE, Kleinschmidt KC, Sharma K, Rapp-Olsson M, et al. Antivenom treatment is associated with fewer patients using opioids after copperhead envenomation. Clin Toxicol. 2019;29(57):25-30.

20. Stratford P. Assessing disability and change on individual patients: a report of a patient specific measure. Physiother Can. 1995;47: 258-63.

21. Hefford C, Abbott JH, Arnold R, Baxter GD. The patient-specific functional scale: validity, reliability, and responsiveness in patients with upper extremity musculoskeletal problems. J Orthop Sports Phys Ther. 2012;42:56-65.

22. Horn KK, Jennings S, Richardson G, Vliet DV, Hefford C, Abbott JH. The patient-specific functional scale: psychometrics, clinimetrics, and application as a clinical outcome measure. J Orthop Sports Phys Ther. 2012;42:30-42.

23. Westaway MD, Stratford PW, Binkley JM. The patient-specific functional scale: validation of its use in persons with neck dysfunction. J Orthop Sports Phys Ther. 1998;27:331-8.

24. Mannberg Bäckman S, Stråt S, Susanne Ahlström S, Brodin N. Validity and sensitivity to change of the patient specific functional scale used during rehabilitation following proximal humeral fracture. Disabil Rehabil. 2016;38:487-92.

25. Koehorst MLS, van Trijffel E, Lindeboom R. Evaluative measurement properties of the patient-specific functional scale for primary shoulder complaints in physical therapy practice. J Orthop Sports Phys Ther. 2014;44:595-603.

26. Anderson VE, Burnham RI, Gillman SM, Daugherty CA, Gerardo CJ, Lavonas EJ. Validation of patient specific functional scale in copperhead snakebite patients. Clin Toxicol. 2015;53:649.

27. Gerardo CJ, Vissoci JRN, Anderson VE, Sharma K, Kleinschmidt $\mathrm{KC}$, Mullins ME, et al. The validity, reliability and minimal clinically important difference of the patient specific functional scale in snake envenomation. Toxicon. 2018;150:352-6.

28. Theophanous RG, Vissoci JRN, Anderson VE, Lavonas EJ, Gerardo CJ. Telephone Administration of the Patient Specific Functional Scale (PSFS): a valid, reliable, and patient-oriented outcome in global snakebite research. Am J Trop Med Hyg. 2017;97(suppl 5):157-8.

Publisher's Note Springer Nature remains neutral with regard to jurisdictional claims in published maps and institutional affiliations. 